\title{
Water on $\operatorname{Pt}(111)$ : the importance of proton disorder
}

\author{
Luigi Delle Site and Luca M. Ghiringhelli \\ Max-Planck-Institut für Polymerforschung, \\ Ackermannweg 10, D-55128 Mainz, Germany \\ Oliviero Andreussi \\ Scuola Normale Superiore, P. ${ }^{z z a}$ dei Cavalieri 7, 56100 Pisa, Italy \\ Davide Donadio \\ Computational Science, Department of Chemistry and Applied Biosciences ETH Zurich, \\ USI-Campus, via Giuseppe Buffi 13, CH-6900 Lugano, Switzerland \\ Michele Parrinello \\ Computational Science, Department of Chemistry and Applied Biosciences ETH Zurich, \\ USI-Campus, via Giuseppe Buffi 13, CH-6900 Lugano, Switzerland and \\ Scuola Normale Superiore, P.zza dei Cavalieri 7, 56100 Pisa, Italy
}

(Dated: October 7, 2018)

\begin{abstract}
The structure of a water adlayer on $\mathrm{Pt}(111)$ surface is investigated by extensive first principle calculations. Only allowing for proton disorder the ground state energy can be found. This results from an interplay between water/metal chemical bonding and the hydrogen bonding of the water network. The resulting short O-Pt distance accounts for experimental evidences. The novelty of these results shed a new light on relevant aspects of water-metal interaction.
\end{abstract}

PACS numbers: $68.43 . \mathrm{Bc}, 73.20 . \mathrm{Hb}, 68.43 . \mathrm{Fg}$ 
The investigation of the interaction of water with solid metal surfaces is of extremely high interest in surface chemistry, catalysis and many other technological applications and is being carried out extensively by means of experiments and theoretical calculations[1, 2]. In particular, much attention has been devoted to the initial stages of the wetting of transition metal surfaces [3, 4, 5], which occurs through the adsorption of monomers or small clusters, and to the structure and stability of very thin water overlayers on $\mathrm{Ru}(1000)$ [6], $\operatorname{Rh}(111)$ [7], $\mathrm{Pt}(111)$ [8, 9] and $\mathrm{Cu}(110)$ [10]. In spite of these important efforts, the determination of the stable structure of water overlayers is in several cases still controversial, as experiments cannot provide conclusive information at the atomistic level. On metals with low proton affinity, as in the cases of $\mathrm{Pt}, \mathrm{Ni}$ and $\mathrm{Cu}$, water arranges in an undissociated hexagonal pattern [11], while on $\mathrm{Ru}(1000)$ a half-dissociated monolayer structure has been demonstrated to be energetically favored [6, 12].

The interaction of water monomers and small clusters on $\mathrm{Pt}(111)$ is by far the best studied [3, 13]. Water is adsorbed at the top site and the bond with the substrate is mainly due to the interaction of the unoccupied $5 d$ states of Pt atoms with the $1 b_{1}$ occupied molecular state of water. Nevertheless, the periodic adlayer can assume three different bilayer arrangements, namely $(\sqrt{39} \times \sqrt{39}) R 16.1^{\circ}$ (RT39), $(\sqrt{37} \times \sqrt{37}) R 25.3^{\circ}$ (RT37) and $(\sqrt{3} \times \sqrt{3}) R 30^{\circ}$ (RT3) [14, 15, 16], with competing adsorption energies [5]. However ab initio calculations showed that the RT39 is the most energetically favored structure [5, 17], the RT3 has been identified in low energy electron diffraction [18] and STM experiments [19]. In addition, the difference in the adsorption energy between the RT3, RT37 and RT39 structures of the wetting layer vanishes to zero as the water coverage increases [5]. Partially dissociated $\left(\mathrm{OH}+\mathrm{H}_{2} \mathrm{O}\right)$ structures have only been observed in connection to water production reaction [20] and theoretically studied [21, 22]. Given this, it is clear that one structure prevails on another as a function of the kinetics and the thermodynamic conditions of the experiments.

While most of the experimental evidences are well described by the remarkable wealth of theoretical results in literature, recent XAS and XPS results indicate a water layer distance to the substrate $(2.4 \AA)$ by far shorter than the theoretically predicted one [9]. Indeed, the previously reported theoretical studies predict that the most stable water configurations is a bilayer, where the shortest Pt-O distance $(2.8 \AA)$ is the result of a weak water-platinum bond. In this configuration the water network is strongly bound and it is left essentially unchanged by the interaction with the metal substrate. This discrepancy between experimental evidence 
and theoretical results is still an open problem and in this Letter we address this issue via a computational approach. We use a large unit cell, since it allows for a less biased search of the lowest energy structure as underlined in Ref.[23], and investigate the possible role of proton disorder which is known to be essential to stabilize not only bulk crystals [33], but also crystalline films [24]; moreover it has been already supposed to play an important role in determining the wetting structures on transition metals surfaces [7, 20]. We show that for this kind of system the proton disorder is essential to understand the ground state structure. In addition, for the fully proton disordered system, some of the water molecules form a strong bond with the substrate leading to a short bonding distance $(2.3 \AA)$. The formation of these strong bonds leads to a weakening of the hydrogen-bond network and the remaining molecules move to a larger distance (3.1-3.5 $\AA$ ). The resulting picture agrees with the experimental findings and allows for an interpretation of the adsorption mechanism.

We use the DFT based finite-electronic temperature method (FEMD) [25] implemented in the plane-wave based CPMD code [26]. In this method the electron density and the HellmanFeynman forces are determined via a subspace diagonalization of the high temperature
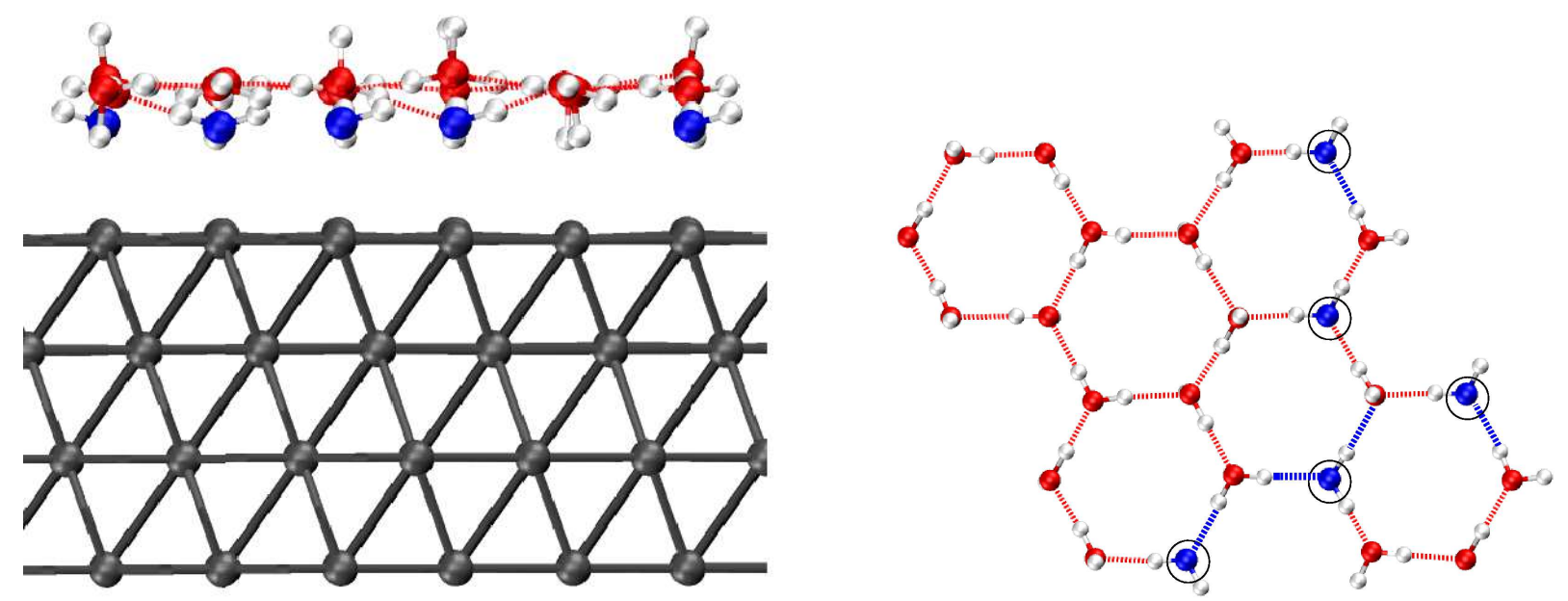

FIG. 1: (Side and top views of the proton disordered $\mathrm{H}_{m i x}$ configuration. The five Pt atoms bonded to the oxygens are raised by $0.1 \AA$ with respect to the average $z$-position of the remaining 31 first layer Pt atoms. The five bonded oxygen atoms (circled in the top view) stay at $2.33 \AA$ from the closest Pt atoms, while the rest of the water network lays 3.1-3.4A far from the surface. Hydrogen bonds are drown in red (blue) when the O-O distance is smaller (greater) than $3.0 \AA$ (see text). 
electron density matrix. The subspace is expanded in a plane-wave basis set that in our set-up is cutoff at $60 \mathrm{Ry}$, a value which, according to our previous work [4], is sufficient to describe satisfactorily the molecule/metal interaction. We use pseudopotentials generated according to Troullier-Martins [27] scheme; all the pseudopotentials were accurately tested to reproduce bulk and surface properties of the metal, and correct structural properties for the molecule. We use the PBE [28] generalized gradient corrected functional. The prototype system consists of a (111) surface of platinum represented by 3 and 4 layers (as explained later on regarding the geometry optimization procedure) and a layer of water composed of 24 molecules. We employ a $6 \times 6$ supercell of hexagonal symmetry with the cell dimension in the direction perpendicular to the surface equal to $25.5 \AA$ so that the thickness of the vacuum between the water molecules and the bottom layer of the image slab of $\mathrm{Pt}(111)$ is equal to about $15 \AA$; due to the extended size of the box, we performed the calculations at the $\Gamma$-point approximation. Geometry optimizations, using the BFGS algorithm, were done first with only 3 layers until the maximum component of the ionic forces was below $2 \times 10^{-3}$ atomic units, then we add a fourth layer and further optimize the structure until the convergence of the root-mean square force was below $10^{-3}$ atomic units, the maximum component of the ionic force was at least below $3 \times 10^{-3}$ and the energy changes were below $0.01 \mathrm{eV}$, i.e. about $10^{-4} \mathrm{eV}$ per molecule into the adsorption energy of water.

Five different proton arrangements are considered: a fully proton-ordered configuration $\left(\mathrm{H}_{\text {down }}^{\text {ord }}\right)$, similar to the ones already studied in literature, two in-plane proton-disordered structures $\left(\mathrm{H}_{\text {up }}\right.$ and $\left.\mathrm{H}_{\text {down }}\right)$ and two fully proton disordered structures, one dissociated $\left(\mathrm{H}_{\text {diss }}\right)$ and one undissociated $\left(\mathrm{H}_{m i x}\right)$. Following the convention of previous works [29] we define $\mathrm{H}_{u p}$ and $\mathrm{H}_{\text {down }}$ as the configuration where the hydrogen atoms not participating to hydrogenbonds point toward the vacuum or metal surface, respectively. The initial configuration of the water film is the one of an ideal (0001) bilayer of hexagonal ice stretched in the $x y$ plane so to match the surface lattice parameter of the metal. In the case of Pt this mismatch is $\sim 8 \%$. The proton disordered configurations have been generated by a Montecarlo method [30], so to minimize either the global or the in-plane dipole moment. Obviously the $z$ component of the dipole moment can be set to zero only if there is no constraint on the number of protons pointing toward the metal surface: in this case we have the so called $\mathrm{H}_{m i x}$ film. In the initial configurations the water molecules are on the top sites of the platinum surface, the vertical separation between the oxygen atoms in the water bilayer is $0.6 \AA$, and 
the lower layer is $2.6 \AA$ apart from the metal slab. In the partially dissociated configuration $\left(\mathrm{H}_{\text {diss }}\right)$, the dissociated protons sit at the free top sites. In Fig. 1 the final geometry of the $\mathrm{H}_{\text {mix }}$ system is reported; the geometries of the other systems are those reported in Ref. [2].

The total adsorption energies per molecule are computed as $E_{a d s}=\left(E_{t o t}-E_{P t}-\right.$ $\left.24 E_{H_{2} O}\right) / 24$, where $E_{t o t}$ is the energy of the optimized Pt+water film system, $E_{P t}$ the energy of the bare Pt surface and $E_{\mathrm{H}_{2} \mathrm{O}}$ the energy of a water molecule. The values are reported in Tab. [ and show very good agreement with those of Ref. [29] as for the $\mathrm{H}_{\text {down }}^{\text {ord }}$; moreover the $\mathrm{H}_{\text {mix }}$ results the most stable. In order to characterize the tendency of the hydrogen bonding network to become less tight as a result of the chemical interaction with the surface, we monitor the number of "strong" hydrogen bonds. An H-bond is defined as "strong" when the O-O distance is smaller than $3.0 \AA$. In the initial configurations this distance is $2.98 \AA$.

In all the undissociated structures, but $\mathrm{H}_{m i x}$, the $\mathrm{H}$-bond network stiffens during the optimization. All the H-bonds shorten, so to recover the ideal O-O distance $(\sim 2.78 \AA)$. Since the underlying metal surface act as a topological confinement, this process (i.e. O-O shortening) is achieved via a reduction of the vertical spacing of the bilayer (initially as long as $0.9 \AA$ ). The water bilayers relaxes at a rather large distance from the Pt surface, with which it does not interact chemically, as confirmed by the charge density plot in Fig,2. In $\mathrm{H}_{\text {diss }}$ both the $\mathrm{OH}$ groups and the undissociated $\mathrm{H}_{2} \mathrm{O}$ bind chemically to the metal surface and form a H-bond pattern where strong (O-O distance: $2.6 \AA)$ and very elongated $(3.1 \AA)$ H-bonds alternate. This picture, confirmed by experiments [31] and previous calculations [32], suggest a competition between the chemical O-Pt bonding and the strength of the H-bond network.

This balance determines the adsorption mechanism of the $\mathrm{H}_{m i x}$ adlayer, which is by far the most stable of the configurations investigated. Due to the full proton disorder, the system is less constrained. The optimal balance is found by sacrificing some of the hydrogenbond energy of the two dimensional water adlayer network (top panel of Fig. 3) and gaining chemical energy with the formation of stronger water-metal bonds. Although the periodicity constraints are relaxed, the water molecules maintain the initial ordered arrangement in the $x y$ plane, while the distribution of vertical heights is clearly bimodal (bottom panel of Fig. 3). The majority of water molecules (80\%) form a flat overlayer where the Pt-O distance is distributed between 3.2 and $3.5 \AA$, while the flat water molecules of the bottom layer bind tightly to $\mathrm{Pt}$ atoms at a distance of $\sim 2.3 \AA$. The electronic density profiles for the 

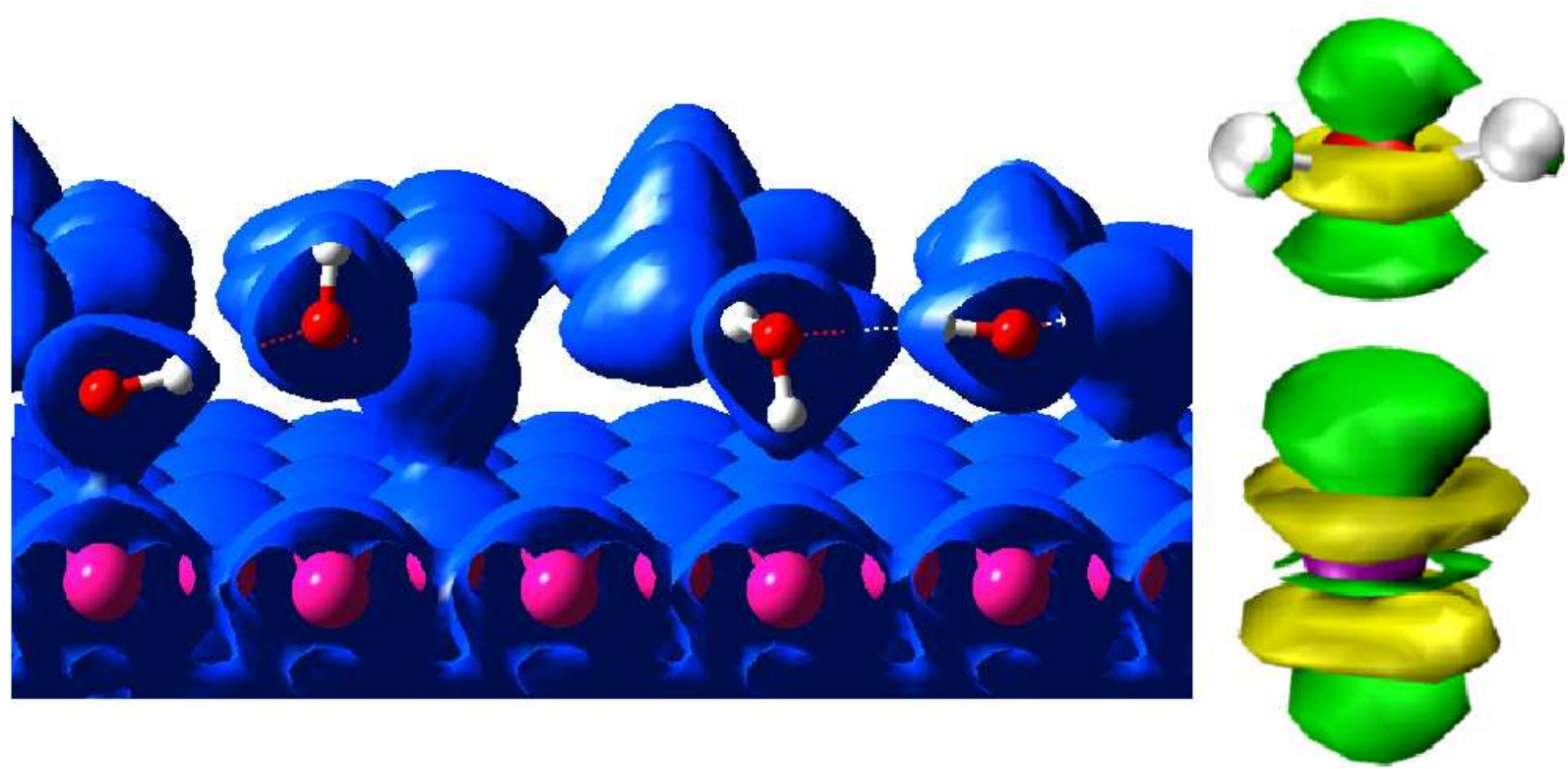

FIG. 2: Electron density profiles for the $\mathrm{H}_{m i x}$ case. The four possible configurations of water molecules are shown in the left panel (the iso-surface level is $0.270 \mathrm{e} / \AA^{3}$ ). From left to right: a flat water molecule in the bottom layer forming a $\mathrm{O}-\mathrm{Pt}$ bond, a water molecule with a hydrogen atom pointing to the vacuum, a water molecule forming a hydrogen bond with the surface and a flat water molecule in the top layer displaying no interaction with the surface. Right panel: electron density difference maps for one of the flat water molecules in the bottom layer, adsorbed via O-Pt bond. The green (yellow) surface indicates depletion (accumulation) of electronic density of 0.036 $e / \AA^{3}$. Note that this snapshot compares very well to the monomer adsorption electron density difference maps shown in Ref. [3].

latter molecules (see the right panel in Fig. 2) shows the formation of chemical bonds with the surface which are much stronger than in the other structures. Moreover the electron density difference of the Pt-Me bonding for the $\mathrm{H}_{m i x}$ case (left panel in Fig. 2) shows the strong chemical bond to be almost similar to that of an isolated monomer [3], i.e. the ideal conformation for the molecular adsorption. As a chemical bond is established between metal and oxygen atoms the hydrogen bonding of the water adlayer is weakened, as proven by the elongation of a number of hydrogen bonds occurring during the optimization of the $\mathrm{H}_{\text {mix }}$ system (Fig. 3).

In order to provide further evidence to the interplay between Pt-O chemical bond and the stiffness of the hydrogen bonding network in the $\mathrm{H}_{m i x}$ system, we substituted $\mathrm{Pt}$ with $\mathrm{Ag}$ 


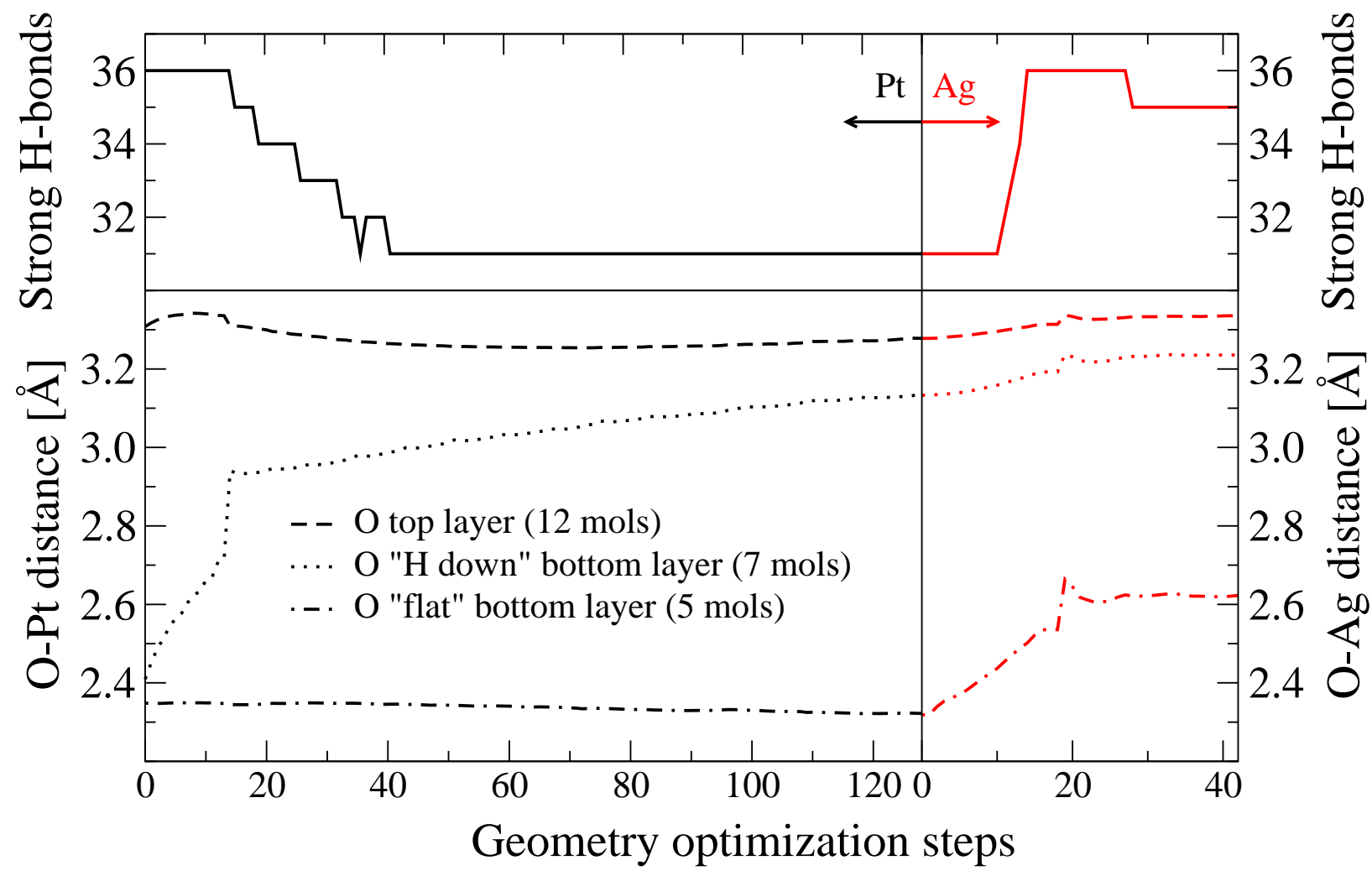

FIG. 3: Top panels: the number of "strong" hydrogen bonds during the geometry optimization process for the $\mathrm{H}_{m i x}$ case on $\mathrm{Pt}(\mathrm{Ag})$ surface is reported on the left (right) side. Bottom panels: Evolution of the distances from the metal surface of the water molecules for the $\mathrm{H}_{\text {mix }}$ configuration. In the left (right) panel we show the distance from the $\mathrm{Pt}(\mathrm{Ag})$ surface of the oxygen of the top water layer (dashed line), of the oxygen of the $\mathrm{H}_{\text {down }}$ water molecules in the bottom layer (dotted line), and (dashed-dotted line) of the "flat" water molecules, i.e. those circled in Fig. 1.

[34], which has a substantially smaller affinity to the adsorption of water [3]. In this manner the metal-oxygen chemical bond is weakened, and performing the geometry optimization we observe the up-shifting of the previously chemisorbed flat $\mathrm{H}_{2} \mathrm{O}$ molecules and the restoration of a tight H-bond network (Fig. 3). The calculated adsorption energy of $-0.46 \mathrm{eV}$ is in good agreement with the ones reported for proton ordered systems in Ref. [29]. This means that for metal surfaces with low water affinity the issue of proton disorder is not relevant.

While providing novel insights about the adsorption mechanism, it is also remarkable that the $\mathrm{H}_{m i x}$ on $\mathrm{Pt}$ is the only configuration that accounts for the x-ray absorption/photoemission spectroscopy measurements in [9], indicating a relatively strong Pt-O bonding, with a layer of oxygen atoms $2.3-2.4 \AA$ far from the surface. It must be noticed 
that, in none of the other undissociated structures considered in this work or in the many previously reported calculations [5, 8, 9, 29], such a short Pt-O bond has been observed.

In conclusion, we have performed an extensive DFT study of the structure and bonding of water adlayers on $\operatorname{Pt}(111)$ surface, where, in particular, we have investigated the role of the proton disorder. We have found that on this particular surface the most stable adlayer is an undissociated configuration with fully proton disorder arrangement. The higher stability of the $\mathrm{H}_{\text {mix }}$ configuration is given by the interplay between the Pt-water chemical bonding and the capability of the water adlayer to preserve its hydrogen-bond network intact, though weakened. We provide the evidence that the main features of the mixed layer/metal interaction cannot be extrapolated from the study of homogeneously oriented (all down or all up) layers. In this sense, this study sheds new light on the nature of the mechanism of interaction of water on metal substrates and thus opens new paths of experimental and theoretical investigations into the field.

L.D.S. acknowledges the RZG of the Max Planck Society for the computational resources, L.M.G. the financial support of the von Humboldt Foundation. Useful suggestions from K. Kremer are acknowledged.

\begin{tabular}{lccccc}
\hline \hline & $E_{a d s}$ & O-H-Pt & O-Pt & H-O-Pt & OH-Pt \\
\hline $\mathrm{H}_{\text {down }}^{\text {row }}$ & -0.49 & 3.1 & 2.8 & - & - \\
$\mathrm{H}_{\text {down }}$ & -0.50 & 3.2 & 3.1 & - & - \\
$\mathrm{H}_{\text {up }}$ & -0.48 & - & 2.9 & 3.5 & - \\
$\mathrm{H}_{\text {mix }}$ & -0.53 & 3.2 & $2.3 / 3.2$ & $3.3 / 3.5$ & - \\
$\mathrm{H}_{\text {diss }}$ & -0.29 & - & 2.2 & - & 2.1 \\
\hline \hline
\end{tabular}

TABLE I: The adsorption energies (in eV) and the distances between oxygen and platinum (in $\AA$ ) are here reported for the 5 systems we studied. The labels of the columns have to be interpreted as follows: O-H-Pt the water molecule has a proton that points toward the $\mathrm{Pt}$ surface, $\mathrm{O}-\mathrm{Pt}$ the water molecule is flat with respect to the metal surface, H-O-Pt the water molecule has a proton that points toward the vacuum, $\mathrm{OH}-\mathrm{Pt}$ refers to a dissociated $\mathrm{OH}$ group. 
[1] C. D. Taylor and M. Neurock, Current Opinion in Solid State and Material Science 9, 49 (2006).

[2] A. Michaelides, Appl. Phys. A 85, 415 (2006).

[3] A. Michaelides, V. A. Ranea, P. L. de Andres, and D. A. King, Phys. Rev. Lett. 90, 216102 (2003).

[4] D. Sebastiani and L. Delle Site, J. Chem. Theory Comput. 1, 78 (2005).

[5] S. Meng, E. G. Wang, and S. Gao, Phys. Rev. B 69, 195404 (2004).

[6] P. J. Feibelman, Science 295, 99 (2002).

[7] P. J. Feibelman, Phys. Rev. Lett. 90, 186103 (2003).

[8] S. Meng, L. F. Xu, E. G. Wang, and S. Gao, Phys. Rev. Lett. 89, 176104 (2002).

[9] H. Ogasawara, B. Brena, D. Nordlund, M. Nyberg, A. Pelmenschikov, L. G. M. Pettersson, and A. Nilsson, Phys. Rev. Lett. 89, 276102 (2002).

[10] J. Ren and S. Meng, J. Am. Chem. Soc. 128, 9282 (2006).

[11] D. L. Doering and T. E. Madey, Surf. Sci. 123, 305 (1982).

[12] G. Held and D. Menzel, Surf. Sci. 316, 92 (1994).

[13] M. Morgenstern, T. Michely, and G. Comsa, Phys. Rev. Lett. 77, 703 (1996).

[14] S. Haq, J. Harnett, and A. Hodgson, Surf. Sci. 505, 171 (2002).

[15] A. Glebov, A. P. Graham, A. Menzel, and J. P. Toennies, J. Chem. Phys. 106, 9382 (1997).

[16] L. E. Firment and G. A. Samoraj, Surf. Sci. 84, 275 (1979).

[17] P. J. Feibelman, Phys. Rev. Lett. 91, 059601 (2003).

[18] K. Jacobi, K. Bedurftig, Y. Wang, and G. Ertl, Surf. Science 472, 9 (2001).

[19] M. Morgenstern, J. Muller, T. Michely, and G. Comsa, Z. Phys. Chem. 198, 43 (1997).

[20] C. Clay, S.Haq, and A. Hodgson, Phys. Rev. Lett. 92, 046102 (2004).

[21] G. S. Karlberg, F. E. Olsson, M. Persson, and G. Wahnstroem, J. Chem. Phys. 119, 4865 (2003).

[22] G. S. Karlberg and G. Wahnstroem, Phys. Rev. Lett. 92, 136103 (2004).

[23] P. Vassilev, R. A. van Santen, and M. T. Koper, J. Chem. Phys. 122, 054701 (2005).

[24] O. Andreussi, D. Donadio, M. Parrinello, and A. H. Zewail, Chem. Phys. Lett. 426, 115 (2006). 
[25] A. Alavi, J. Kohanoff, M. Parrinello, and D. Frenkel, Phys. Rev. Lett. 73, 2599 (1994).

[26] CPMD, version 3.10.5, developed by J. Hutter et al., MPI für Festkörperforschung and IBM Zurich Research Laboratory.

[27] N. Troullier and J. L. Martins, Phys. Rev. B 43, 1993 (1991).

[28] J. P. Perdew, K. Burke, and M. Ernzerhof, Phys. Rev. Lett. 77, 3865 (1996).

[29] A. Michaelides, A. Alavi, and D. A. King, Phys. Rev. B 69, 113404 (2004).

[30] V. Buch, P. Sandler, and J. Sadlej, J. Phys. Chem. B 102, 8641 (1998).

[31] G. Held, C. Clay, S. D. Barrett, S. Haq, and A. Hodgson, J. Chem. Phys. 123, 064711 (2005).

[32] T. Jacob and W. A. Goddard III, J. Am. Chem. Soc. 126, 9360 (2004).

[33] The most common polymorphs of ice are indeed proton disordered

[34] Computational details are the same as for the other systems treated here. For Ag we use an LDA pseudopotential previously tested according to the criteria employed in this work. The final O-O distances for $\mathrm{H}_{m i x}$ configuration on Pt, was scaled according to the lattice constant of $\mathrm{Ag}$, and, as a consequence, the same is done when counting hydrogen bonds 